\title{
1 AAV11 permits efficient retrograde targeting of projection neurons
}

2 Zengpeng Han ${ }^{1,2,3}$, Nengsong Luo ${ }^{1,4 \#}$, Jiaxin $\mathrm{Kou}^{1,5}$, Lei $\mathrm{Li}^{1}$, Wenyu Ma ${ }^{1,2}$, Siqi Peng ${ }^{6}$, 3 Zihong $\mathrm{Xu}^{6}$, Wen Zhang ${ }^{6}$, Yuxiang Qiu ${ }^{2,3}$, Yang $\mathrm{Wu}^{1}$, Jie Wang ${ }^{1,2}$, Chaohui Ye ${ }^{1,2}$,

$4 \quad$ Kunzhang $\operatorname{Lin}^{3 *}$, Fuqiang $\mathrm{Xu}^{1,2,3,4,7,8 *}$

${ }^{1}$ Key Laboratory of Magnetic Resonance in Biological Systems, State Key Laboratory of Magnetic Resonance and Atomic and Molecular Physics, National Center for Magnetic Resonance in Wuhan, Wuhan Institute of Physics and Mathematics, Innovation Academy for Precision Measurement Science and Technology, Chinese Academy of Sciences-Wuhan National Laboratory for Optoelectronics, Wuhan, 430071, P.R. China.

${ }^{2}$ University of Chinese Academy of Sciences, Beijing, 100049, P.R. China.

${ }^{3}$ The Brain Cognition and Brain Disease Institute (BCBDI), Shenzhen Key Laboratory of Viral Vectors for Biomedicine, Shenzhen Institute of Advanced Technology, Chinese Academy of Sciences; Shenzhen-Hong Kong Institute of Brain Science-Shenzhen Fundamental Research Institutions, NMPA Key Laboratory for Research and Evaluation of Viral Vector Technology in Cell and Gene Therapy Medicinal Products, Shenzhen, Key Laboratory of Quality Control Technology for Virus-Based Therapeutics, Guangdong Provincial Medical Products Administration, Shenzhen, 518055, P.R. China.

${ }^{4}$ Wuhan National Laboratory for Optoelectronics, Huazhong University of Science and Technology, Wuhan 430074, P.R. China.

${ }_{5}^{5}$ Department of Pathophysiology, Key Lab of Neurological Disorder of Education Ministry, School of Basic Medicine, Tongji Medical College, Huazhong University of Science and Technology, Wuhan, P. R. China.

${ }^{6}$ College of Life Sciences, Wuhan University, Wuhan, P.R. China.

${ }^{7}$ Shenzhen-Hong Kong Institute of Brain Science-Shenzhen Fundamental Research Institutions, Shenzhen, 518055, P.R. China.

${ }^{8}$ Center for Excellence in Brain Science and Intelligence Technology, Chinese Academy of Sciences, Shanghai, 200031, P.R. China.

*Correspondence: kz.lin@siat.ac.cn (K.Lin.); fq.xu@siat.ac.cn (F.Xu.).

\# These authors contributed equally to this work. 


\title{
Highlights
}

1. Naturally occurring AAV serotype capsid exhibits robust retrograde functionality

2. Improved distribution properties and retrograde transport efficiency

3. Can express Cre recombinase or calcium-sensitive functional probe for neural circuits monitoring

4. Can specifically target astrocytes

\begin{abstract}
:
Viral tracers that permit efficient retrograde targeting of projection neurons are powerful vehicles for structural and functional dissections of the neural circuit and for the treatment of brain diseases. Recombinant adeno-associated viruses (rAAVs) are the most potential candidates because they are low-toxic with high-level transgene expression and minimal host immune responses. Currently, some rAAVs based on capsid engineering for retrograde tracing have been widely used in the analysis and manipulation of neural circuits, but suffer from brain area selectivity and inefficient retrograde transduction in certain neural connections. Here, we discovered that the recombinant adeno-associated virus 11 (rAAV11) exhibits potent retrograde labeling of projection neurons with enhanced efficiency to rAAV2-retro in some neural connections. Combined with calcium recording technology, rAAV11 can be used to monitor neuronal activities by expressing Cre recombinase or calcium-sensitive functional probe. In addition, we further showed the suitability of rAAV11 for astrocyte targeting. These properties make rAAV11 a promising tool for the mapping and manipulation of neural circuits and gene therapy of some neurological and neurodegenerative disorders.
\end{abstract}




\section{Keywords:}

Adeno-associated virus 11, Retrograde, Natural serotype capsid, Projection Neurons

\section{Introduction:}

Several major mental diseases caused by structural variation or loss of neural circuits, such as Parkinson's disease, Alzheimer's disease, schizophrenia, autism, etc., have brought a heavy burden to the family and society. At present, there is still a lack of effective prevention and cure methods, and the main reason is that the mechanism of disease-related complex circuit changes is unclear. Analyzing the structure and function of brain neural network is fundamental to revealing the working principle of brain and the mechanism of brain diseases. However, mapping and manipulating functional network connection has become an important challenge due to the lacking of techniques available for targeting projection neurons. While classic neuroanatomical retrograde tracers have been widely used to analysis the architectural connectivity between different brain regions, they cannot deliver genetic cargos for neural activity manipulation and clinical application [1].

Recombinant viral vectors have been developed as valuable tools to address the limitations of traditional retrograde tracers, because they can not only express fluorescent proteins for connection network visualization, but also carry functional probes (e.g., indicators or effectors of neural activity) for the monitoring or manipulation of neural circuits. Many naturally evolved and engineered viruses can transduce projection neuron populations by axon terminal uptake [2, 3], including canine adenovirus-2 (CAV-2), rabies virus (RV), herpes simplex virus (HSV), and lentivirus (LV) among others. Of these, CAV-2 can effectively retrograde label longrange projection neurons $[4,5]$, but allows only moderate levels of transgene expression, presents some toxicity $[6,7]$, and has difficulties in large-scale preparation for clinic applications [8]. While RV and HSV display robust efficiency of retrograde labeling with rapid gene expression, they are not suitable for functional research and gene 
therapy due to high cytotoxicity. [9-14]. Lentiviruses pseudotyped with modified RVG present the improved retrograde transduction ability in rodents and non-human primates $[15,16]$, but have a risk of tumorigenesis due to random integration into the host genome [17]. Thus, developing non-toxic, readily manufactured viral vectors that mediate flexible packaging of different transgenes, retrograde transduction, and highlevel gene expression remains an urgent need.

In this case, the use of capsid engineering adeno-associated virus (AAV) for retrograde labeling becomes a good option because of its safety, non-pathogenic nature, and ability to infect dividing and quiescent cells (e.g., neurons) in vivo [18, 19]. Many engineered AAV capsids for retrograde tracing have been reported, such as AAV2-retro [20], AAV9-retro [21], AAV2-MNM004 [22], AAV9-SLR [23], etc. Among them, AAV2retro was developed by the insertion of a fragment (LADQDYTKTA) from 10-mer peptide libraries between N587 and R588 of the wild-type AAV2 VP1 capsid gene, displaying the most robust retrograde transport, has been extensively used to express fluorescent probes to analyze the structural connections of neural networks and to express functional probes to monitor and manipulate neuronal activities [20]. However, AAV2-retro suffers from brain area selectivity and inefficient retrograde transduction in certain neural connections [24]. And because the retrograde characteristics of these AAV carriers are given through technical modification, it may not be easy to have further improvements.

In addition to the capsid engineering methods such as directed evolution or rational design, a more fundamental way to find a better AAV is to identify new natural serotypes or new characteristics of natural serotypes. The results of this method can be used as a basis for further engineering work, thereby increasing the underlying optimization potential. There are many natural serotypes of AAV, which belong to different pedigrees and have their unique infection characteristics [25-27]. In neuroscience research, the commonly used AAV serotypes are 1, 2, 5, 6, 8, and 9, but low propensity of retrograde transduction hinders their applications in the research of projection network or disease treatment. Here, we developed the recombinant adeno- 
116 associated virus serotype 11 (rAAV11), and found that it exhibits potent retrograde

117 labeling of projection neurons with enhanced efficiency to rAAV2-retro in some neural

118 connections. Combined with calcium recording technology, rAAV11 can be used to

119 monitor neuronal activities by expressing Cre recombinase or calcium-sensitive

120 functional probe. In addition, we further showed the suitability of rAAV11 for astrocyte

121 targeting. These properties make rAAV11 a promising tool for the mapping and

122 manipulation of neural circuits and gene therapy of some neurological and

123 neurodegenerative disorders. As a basis vector, it has considerable potential for further

124 engineering optimization.

\section{Results}

\section{Obtain rAAV11 virus vector based on HEK293T production system}

We have previously established a single baculovirus-insect cell-based large-scale production system (OneBac system) and verified the preparation of rAAV1-13 serotypes $[28,29]$. Although it is conducive to large-scale production, putting all the genetic elements in the common bacmid is not convenient for switching the carried foreign genes. To facilitate laboratory-level verification, we established the AAV packaging method based on the three plasmids. The pAAV2/11 plasmid was obtained by inserting AAV11 Cap sequence into the pAAV2/1 backbone to substitute the AAV1 Cap gene and its efficiency in virus packaging was evaluated in HEK-293 cells. We found that pAAV2/11 plasmid could be used to package high-titer rAAV2/11 with efficiency comparable to rAAV2/9.

\section{rAAV11 mediates efficient neuron transduction with axon terminal absorption}

139 The dorsal striatum, called the caudate-putamen $(\mathrm{CPu})$, includes the caudate and

140 putamen nucleus, which is innervated by dopaminergic neurons from the substantia 141 nigra (SN) pars compacta [30]. It's one of the structures that compose the basal nuclei.

142 Through various pathways, it receives dopaminergic inputs from the ventral tegmental 
area (VTA) and the $\mathrm{SNr}$ and glutamatergic inputs from several regions, including the cortex, hippocampus, amygdala, and thalamus [31-35]. A primary function of the caudate-putamen is to regulate movements at various stages and influence different types of learning. The caudate-putamen also plays a role in degenerative neurological disorders, such as Parkinson's disease [36].

To evaluate the infectious effect of rAAV11, rAAV11-EF1 $\alpha$-EGFP viruses were delivered to $\mathrm{CPu}$ via stereotactic injection $\left[3 \times 10^{9}\right.$ vector genomes $(\mathrm{VG})$ in total $]$ in adult mice and the fluorescence distribution was assessed (Fig.1 A, B). Fig.1 B shows the fluorescence distribution of EGFP expression at the injection site, with a pretty broad spread range. Fig.1 $\mathrm{C}$ shows the spread of the virus towards the anterior side, Fig.1 D shows the spread of the virus to the posterior side, clear neuron morphology can be labeled in several upstream brain areas, such as somatomotor areas (MO), anterior olfactory nucleus (AON), primary somatosensory area (SSp), and mediodorsal nucleus of thalamus (MD), among others. These results suggest that rAAV11 has excellent retrograde labeling capability and can be transported to the cellular body of upstream neurons by axon terminal uptake.

\section{rAAV11 does not exhibit anterograde transsynaptic spread property}

Some AAV serotypes (such as AAV1 and AAV9) exhibit the features of anterograde transsynaptic propagation [37], which can also cause an extensive spread range. To test whether rAAV11 can mediate anterograde transsynaptic spread, rAAV11-hSyn-Cre was injected into primary visual cortex (V1) of Ail4 (CAG promoter-driven and Credependent expression of tdTomato reporter $)$ mice [38] $\left(2 \times 10^{9} \mathrm{VG}\right.$ in total) (Fig.2 A, B). Following a 3 week post-injection, a number of tdTomato fluorescence signals were detected at the injection site, but no tdTomato-expressing cell bodies were observed in the downstream regions known to be directly projected by V1 (Fig.2 B, B1, C, C1), including the superior colliculus (SC) and caudate-putamen $(\mathrm{CPu})$, which had been proved not to project to V1 [39, 40], indicating that the spread of rAAV11 is not caused by the anterograde transsynaptic spread. 
As an effective and practical retrograde viral tracer, rAAV2-retro is widely used in the analysis and manipulation of different types of neural circuitry [41, 42], as well as disease modeling and therapeutic evaluation [43]. To estimate the performance of rAAV11 retrograde tagging, we compared rAAV11 and rAAV2-retro by injecting an equal amount of rAAV2-retro and rAAV11 carrying different fluorescent reporter genes. rAAV11-EF1 $\alpha$-EGFP and rAAV2-retro-EF1 $\alpha$-mCherry are mixed in equal amounts and injected into $\mathrm{CPu}$ (Fig.3), ventral hippocampus (vHPC) (Fig.4) and periaqueductal gray (PAG) (Fig.5), respectively.

At the $\mathrm{CPu}$ injection site, it can be observed that rAAV11 has a more extensive spreading range than rAAV2-retro (Fig.3 B), in the upstream SSp brain area of $\mathrm{CPu}$, both tracers can be detected, but rAAV11 label more cells and tends to infect the fourth layer, while rAAV2-retro prefer to infect the fifth layer (Fig.3 B1 D1), similar infection tendency can also be observed in MO (Fig.3 C1) and MD (Fig.3 D2), still, rAAV11 has a more extensive infection range. In particular, in the orbital area (ORB), there were far more cells labeled with rAAV11 than rAAV2-retro, and rAAV2-retro only labeled a few cells. A similar pattern is illustrated in Figure 4, when injected in the vHPC area (Fig.4 A, B). rAAV11 has excellent retrograde tracing in hippocampal field CA3 (CA3), nucleus of reuniens (RE), endopiriform nucleus dorsal part (EPd), lateral dorsal nucleus of thalamus (LD), and medial septal complex (MSC), while rAAV2-retro label few cells (Fig.4 C1, C2). The periaqueductal gray (PAG) is a critical structure in the propagation and modulation of pain, sympathetic responses, as well as the learning and action of defensive and aversive behaviors [44]. We also tested the ability of rAAV11 and rAAV2-retro to retrogradely label the upstream brain regions of PAG (Fig.5). Although a comparable level of tracing effect can be seen in ectorhinal area (ECT) (Fig.5 C), rAAV11 still shows a better tracing effect in ventromedial hypothalamic nucleus (VMH) and offers a more extensive spread range in PAG. 

mice

Humans and other primates use their hands to engage in tactile discrimination that directs choices and actions. The vast majority of such decisions are based on stimuli which are presented to the sites by hand or hands, the transformation of these information sources dispersed from receptors in distinct plates of the skin into a percept presupposes integration into the central nervous system, SSp played an essential role in this process [45]. As a proof of principle, with Ai14 transgenic mice, we use rAAV11Cre to trace the upstream projection neurons of SSp (Fig.6 A, B). The tdTomato signal is visible in ventral anterior-lateral complex of the thalamus (VAL) and ectorhinal area (ECT) (Fig.6 C).

\section{Using rAAV11 for functional circuit interrogation}

GCaMP is a genetically encoded calcium sensor consisting of a circular GFP, calmodulin (CaM), and a peptide chain (M13), which in its natural conformation shows only poor fluorescence. In the presence of calcium, $\mathrm{CaM}$ undergoes a structural change that entails a rapid increase in fluorescence [46]. GCaMP6m reliably detected single action potentials in neuronal somata and orientation-tuned synaptic calcium transients in individual dendritic spines [47]. The dopamine projection from the ventral tegmental area (VTA) to nucleus accumbens (NAc) is critical for motivation to work for rewards and reward-driven learning [48]. To verify the application of rAAV11 in functional circuit interrogation, the experiment was conducted using a retrogradely induced GCaMP6m expression, using an injection of an rAAV11-Cre vector in the NAc combined with an infusion of a Cre-inducible GCaMP6m vector in the VTA (Fig. 7A) of $\mathrm{C} 57 \mathrm{BL} / 6 \mathrm{~J}$ mice. We set up sucrose solution as a reward in the behavior box of the mice (Fig.7 A, B), when mice licked sugar water to get rewards, VTA neurons projecting to the NAc were activated (Fig.7 C), then the calcium ion signal could be detected (Fig.7 D, E). 
Glial cells are critical responders to central nervous system injury and equal the number of neurons in the adult human brain $[49,50]$. Furthermore, astrocytes are the most abundant glial cell type in the CNS and they play a crucial role in the pathogenesis of spinal cord injury [51]. However, some serotypes of AAV cannot target glial cells, the viral tracers for specifically targeting astrocytes are still lacking. We tested the targeting ability of rAAV11 to astrocytes in dentate gyrus (DG) (Fig.8 A, B). By carrying the EGFP reporter gene initiated by $\mathrm{GfaABC}_{1} \mathrm{D}$ promoter, we observed that the green fluorescent protein and astrocytes have a reasonable co-labeling (Fig.8 C).

\section{Discussion}

Currently, AAV is among the most commonly used viruses for neuroscience research [52] and the leading platform for in vivo delivery of gene therapies [53], have been extensively used as vehicles for gene transfer to the nervous system enabling gene expression and knockdown, gene editing [54, 55], circuit modulation [56, 57], in vivo imaging $[58,59]$, disease model development [60], and the evaluation of therapeutic candidates for the treatment of neurological diseases [18]. Focus on the application of AAV vectors in central nervous system related issues, in addition to the delivery of the gene of interest by in situ injection, the infection types of AAV mainly include across the blood-brain barrier via intravenous injection [61-64], anterograde transsynaptic spread [37], and retrograde labeling by axon terminal absorption [20-23, 65].

There are various natural serotypes of $\mathrm{AAV}$, which belong to different pedigrees and have their unique tropism $[25-27,66]$, this is one of the reasons why AAV is widely used. Our research has tested the rarely used natural serotype AAV11, which capsid has not been artificially modified, and found that it has a highly efficient retrograde effect. AAV2-retro was promoted and applied, and the AAV9-retro [21], AAV2-MNM004 [22], and AAV9-SLR [23] were obtained by engineering modification later, but the effect of 
253 points worth noting in our research: first, the retrograde capability of rAAV11 has been 254 better than rAAV2-retro to a certain extent (Fig.3, Fig.4, Fig.5); second, as a natural 255 serotype, the capsid of AAV11 still has excellent potential for further technical upgrades. 256 In addition, we confirmed that rAAV11 does not have anterograde transsynaptic properties (Fig.2), and can carry functional elements for genetic manipulation (Fig.6, Fig.7).

Especially, we found that rAAV11 can efficiently target astrocytes (Fig.8). Although astrocytes have traditionally been described as playing a supportive role for neurons, they have recently been recognized as active participants in the development and plasticity of dendritic spines and synapses [67]. Astrocyte dysfunction has in recent years proven to be a common crossroads in neurodegenerative disorders such as stroke [68], Alzheimer's disease [69], Parkinson's disease [70] and Huntington's disease [71], alteration in astrocytic glutamate uptake is a core feature of multiple neurodegenerative disorders $[67,72]$, with the in-depth research on astrocytes, rAAV11 may be used as a powerful candidate virus tool for scientific research or gene therapy.

Based on the above nervous system infection tropism, rAAV11 can become a new engineering target for virus vector designers. AAV capsid engineering offers great potential and the successful promotion of mutation vectors such as AAV2-retro benefits from this. There are at least four main approaches currently used towards this: directed evolution [20, 22, 61, 62, 73, 74], rational design [75-79], in silico evolutionary lineage analysis [80, 81], and chemical conjugation [82-88]. The "natural occurring" of AAV11 capsid has become one of its advantages, with reference to the previous experience of AAV capsid engineering, it might be possible to obtain a better AAV11 mutant version through artificial upgrades, whether in retrograde labeling or astrocyte targeting. 
Material and Methods

\section{Plasmids construction and virus manufacturing}

280 To obtain the pAAV2/11 plasmid, AAV11 Cap sequence retrieved on Genbank 281 (accession number: AY631966.1) was synthesized as a template for amplifying AAV11 Cap fragment using PrimeSTAR HS DNA Polymerase (Takara, R040A), After being digested by using SwaI and AgeI (New England Biolabs) restriction endonucleases, the AAV11 Cap fragment was inserted into pAAV-RC2/1 vector (Addgene, \#112862) by T4 DNA ligase (New England Biolabs, M0202M), then the ligation product was transformed into Stbl3 chemically competent $E$. coli, the positive clone was picked after PCR identification and the plasmid was extracted to obtain pAAV2/11.

The plasmids carrying the foreign gene of interest (e.g., pAAV-hSyn-Cre-WPRE-pA, pAAV-EF1 $\alpha$-EGFP-WPRE-pA, pAAV-EF1 $\alpha$-mCherry-WPRE-pA, pAAV-EF1 $\alpha$-DIOGCaMP6m-WPRE-pA or pAAV-GfaABC ${ }_{1}$ D-EGFP-WPRE-pA) with pAAV-RC2/11 (or pAAV-RC2-retro or pAAV-RC9) and pAdDeltaF6 (Addgene plasmid \#112867) were co-transfected into HEK-293T cells at the molecular ratio of 1:1:1, samples were harvested at 72 hours post-transfection and purified by iodixanol gradient ultracentrifugation $[29,89,90]$. The purified rAAVs were titred by qPCR using the iQ SYBR Green Supermix kit (Bio-Rad, 1708884) and diluted to $1.0 \times 10^{13} \mathrm{VG} / \mathrm{mL}$. All viral vectors were aliquoted and stored at $-80^{\circ} \mathrm{C}$ until use.

\section{Research animals}

298 Adult male (8-10 weeks old) C57BL/6J mice (Hunan SJA Laboratory Animal 299 Company) and Ai14 transgenic mice (The Jackson Laboratory) were used for experiments. The mice were housed in the appropriate environment with a 12/12-h

301 light/dark cycle, water and food were supplied ad libitum. All the surgical and experimental procedures were performed following the guidelines formulated by the

303 Animal Care and Use Committee of Innovation Academy for Precision Measurement 
Briefly, the mice were deeply anaesthetized using $1 \%$ pentobarbital intraperitoneally (i.p., $50 \mathrm{mg} / \mathrm{kg}$ body weight). The stereotactic injection coordinates were selected according to Paxinos and Franklin's The Mouse Brain in Stereotaxic Coordinates, 4th edition [91], animals were placed on a stereotactic frame (RWD, 68030). A small volume of the virus was injected into the $\mathrm{CPu}$ (anterior-posterior-axis (AP) $+0.75 \mathrm{~mm}$

311 from bregma, medial-lateral-axis (ML) $\pm 2.00 \mathrm{~mm}$, and dorsal-ventral-axis (DV) -2.00 $\mathrm{mm}), \mathrm{V} 1$ (AP $-3.90 \mathrm{~mm}$ from bregma, $\mathrm{ML} \pm 2.6 \mathrm{~mm}$, and DV $-1.30 \mathrm{~mm})$, vHPC (AP $3.16 \mathrm{~mm}$ from bregma, $\mathrm{ML} \pm 2.95 \mathrm{~mm}$, and $\mathrm{DV}-4.10 \mathrm{~mm}$ ), PAG (AP $-4.00 \mathrm{~mm}$ from bregma, $\mathrm{ML} \pm 0.26 \mathrm{~mm}$, and DV $-2.60 \mathrm{~mm}$ ), SSp (AP $+0.50 \mathrm{~mm}$ from bregma, ML $\pm 3.00 \mathrm{~mm}$ and DV $-2.00 \mathrm{~mm}$ ), VTA (AP $-3.20 \mathrm{~mm}$ from bregma, $\mathrm{ML} \pm 0.45 \mathrm{~mm}$, and $\mathrm{DV}-4.30 \mathrm{~mm})$, NAc (AP $+1.50 \mathrm{~mm}$ from bregma, $\mathrm{ML} \pm 1.10 \mathrm{~mm}$, and $\mathrm{DV}-4.60 \mathrm{~mm})$, and $\mathrm{DG}(\mathrm{AP}-2.15 \mathrm{~mm}$ from bregma, $\mathrm{ML} \pm 1.30 \mathrm{~mm}$, and $\mathrm{DV}-2.00 \mathrm{~mm})$, respectively, using a pulled glass capillary with stereotaxic injector (Stoelting, 53311) at a slow rate of $0.03 \mu \mathrm{L} / \mathrm{min}$. After the injection was completed, the capillary was left for an additional 10 minutes before slowly being withdrawn completely. After surgery, animals were allowed to recover from anaesthesia under a heating pad. At three weeks post-injection, the mice were sacrificed. Mice were deeply anesthetized with an overdose of $5 \%$ chloral hydrate and transcardially perfused ice-cold PBS (pH 7.4) followed by $4 \%$ paraformaldehyde. After overnight post fix in 4\% paraformaldehyde solution, brains were dehydrated in $30 \%$ sucrose solution for one day.

\section{Slice preparation, immunohistochemistry, and imaging}

327 Slice preparation and imaging were completed according to the previously reported methods [21]. Coronal sections $(40 \mu \mathrm{m})$ were cut on a microtome (Thermo Fisher

329 Scientific), collected in anti-freeze fluid, and stored at $-20{ }^{\circ} \mathrm{C}$ for further use. For staining the GFAP, the primary antibody used were goat anti-GFAP (1:800, Abcam),

331 amplified with secondary antibody rabbit anti-goat IgG conjugated with Cy3 (1:400, Jackson), and fixed slices stained with DAPI (1:4000), then washed with PBS three 
333 times, followed by sealing with $70 \%$ glycerol. Imaging was performed using the

334 Olympus VS120 Slide Scanner microscope (Olympus).

\section{Imaging of neuronal population activity in vivo following delivery of GCaMP6m}

rAAV11-hSyn-Cre $\left(2 \times 10^{9} \mathrm{VG}\right)$ and rAAV9-DIO-GCaMP6m $\left(2 \times 10^{9} \mathrm{VG}\right)$ was injected into the NAc and VTA, respectively, and optical fiber (core diameter: $200 \mu \mathrm{m}$, numerical aperture: 0.37, Inper, China) was implanted in VTA. Mice had visually identifiable GCaMP6m-expressing cells in VTA two weeks post-injection. Before recording, the mice were handled for 3-5 min at least 3 days and then were habituated to the fiber patch cord and a chamber $(20 \times 20 \times 22 \mathrm{~cm})$ for $10 \mathrm{~min}$. All mice were water deprived for $24 \mathrm{~h}$ before placed in the chamber, which was equipped with a cup filled with $5 \%(\mathrm{w} / \mathrm{v})$ sucrose solution. Then the mice were tested when searching for sucrose rewards. Calcium transients during behavior were recorded by exciting GCaMP6m at $470 \mathrm{~nm}$ using the fiber photometry system (ThinkerTech, Nanjing, China). Data were analysed using MATLAB (MathWorks) code and Prism (GraphPad software).

\section{Acknowledgments}

We are grateful to Liting Luo and Lingling $\mathrm{Xu}$ (Core Facility Center, Innovation Academy for Precision Measurement Science and Technology, Chinese Academy of Sciences) for technical assistance with centrifugation and microscope imaging. Schematic diagrams in the pictures of this article were created with BioRender.com. This work was supported by the National Natural Science Foundation of China (31830035, 31771156, 21921004), the Key-Area Research and Development Program of Guangdong Province (2018B030331001), the Strategic Priority Research Program of the Chinese Academy of Sciences (XDB32030200) and the Shenzhen Key Laboratory of Viral Vectors for Biomedicine (ZDSYS20200811142401005). 


\section{Authors' contributions}

$360 \mathrm{KL}$ and FX contributed to the study idea and design; FX, CY and JW contributed to funding acquisition and resources; ZH, NL, JK, LL, WM, SP, ZX, WZ and YQ performed the experiments and data acquisition; ZH, NL, KL and YW accomplished data analysis; ZH, KL and FX drafted the manuscript, and contributed to review and editing. All authors read and approved the final manuscript.

\section{Declaration of interests}

367 The authors declare no competing interests.

\section{References}

370 1. Li SJ, Vaughan A, Sturgill JF and Kepecs A. A Viral Receptor Complementation Strategy to Overcome CAV-2 Tropism for Efficient Retrograde Targeting of Neurons. Neuron.

2. Callaway EM. Transneuronal circuit tracing with neurotropic viruses. Curr Opin Neurobiol.

3. Larsen DD, Wickersham IR and Callaway EM. Retrograde tracing with recombinant rabies

4. Soudais C, Laplace-Builhe C, Kissa K and Kremer EJ. Preferential transduction of neurons by canine adenovirus vectors and their efficient retrograde transport in vivo. Faseb Journal. 2001;15(10):2283-+.

5. Junyent $\mathrm{F}$ and Kremer EJ. CAV-2-why a canine virus is a neurobiologist's best friend. Current

6. Piersanti S, Astrologo L, Licursi V, Costa R, Roncaglia E, Gennetier A, et al. Differentiated neuroprogenitor cells incubated with human or canine adenovirus, or lentiviral vectors have adenovirus-mediated gene transfer. J Virol. 2000;74(1):505-12. 
9. Vercelli A, Repici M, Garbossa D and Grimaldi A. Recent techniques for tracing pathways in the central nervous system of developing and adult mammals. Brain Res Bull. 2000;51(1):1128.

10. Katz LC. Local circuitry of identified projection neurons in cat visual cortex brain slices. J Neurosci. 1987;7(4):1223-49.

11. Wickersham IR, Finke S, Conzelmann KK and Callaway EM. Retrograde neuronal tracing with a deletion-mutant rabies virus. Nat Methods. 2007;4(1):47-9.

12. Suzuki L, Coulon P, Sabel-Goedknegt EH and Ruigrok TJ. Organization of cerebral projections to identified cerebellar zones in the posterior cerebellum of the rat. J Neurosci. 2012;32(32):10854-69.

13. Zhu X, Lin K, Liu Q, Yue X, Mi H, Huang X, et al. Rabies Virus Pseudotyped with CVS-N2C Glycoprotein as a Powerful Tool for Retrograde Neuronal Network Tracing. Neurosci Bull. 2020;36(3):202-216.

14. Su P, Ying M, Han Z, Xia J, Jin S, Li Y, et al. High-brightness anterograde transneuronal HSV1 H129 tracer modified using a Trojan horse-like strategy. Molecular Brain. 2020;13(1).

15. Kato S, Kobayashi K, Inoue K, Kuramochi M, Okada T, Yaginuma H, et al. A lentiviral strategy for highly efficient retrograde gene transfer by pseudotyping with fusion envelope glycoprotein. Hum Gene Ther. 2011;22(2):197-206.

16. Tanabe S, Inoue KI, Tsuge H, Uezono S, Nagaya K, Fujiwara M, et al. The use of an optimized chimeric envelope glycoprotein enhances the efficiency of retrograde gene transfer of a pseudotyped lentiviral vector in the primate brain. Neurosci Res. 2017;120:45-52.

17. Themis M, Waddington SN, Schmidt M, von Kalle C, Wang Y, Al-Allaf F, et al. Oncogenesis following delivery of a nonprimate lentiviral gene therapy vector to fetal and neonatal mice (vol 12, pg 763, 2005). Molecular Therapy. 2006;13(4):830-830.

18. Hocquemiller M, Giersch L, Audrain M, Parker S and Cartier N. Adeno-Associated Virus-Based Gene Therapy for CNS Diseases. Hum Gene Ther. 2016;27(7):478-96.

19. Choudhury SR, Hudry E, Maguire CA, Sena-Esteves M, Breakefield XO and Grandi P. Viral vectors for therapy of neurologic diseases. Neuropharmacology. 2017;120:63-80.

20. Tervo DG, Hwang BY, Viswanathan S, Gaj T, Lavzin M, Ritola KD, et al. A Designer AAV Variant Permits Efficient Retrograde Access to Projection Neurons. Neuron. 2016;92(2):372382.

21. Lin K, Zhong X, Li L, Ying M, Yang T, Zhang Z, et al. AAV9-Retro mediates efficient transduction with axon terminal absorption and blood-brain barrier transportation. Mol Brain. 2020;13(1):138.

22. Davidsson M, Wang G, Aldrin-Kirk P, Cardoso T, Nolbrant S, Hartnor M, et al. A systematic capsid evolution approach performed in vivo for the design of AAV vectors with tailored properties and tropism. Proc Natl Acad Sci U S A. 2019. 
23. Commisso B, Ding L, Varadi K, Gorges M, Bayer D, Boeckers TM, et al. Stage-dependent remodeling of projections to motor cortex in ALS mouse model revealed by a new variant retrograde-AAV9. Elife. 2018;7.

24. Zhu XT, Lin KZ, Liu Q, Yue XP, Mi HJ, Huang XP, et al. Rabies Virus Pseudotyped with CVSN2C Glycoprotein as a Powerful Tool for Retrograde Neuronal Network Tracing. Neuroscience Bulletin. 2020;36(3):202-216.

25. Gao G, Vandenberghe LH, Alvira MR, Lu Y, Calcedo R, Zhou X, et al. Clades of Adenoassociated viruses are widely disseminated in human tissues. J Virol. 2004;78(12):6381-8.

26. Mietzsch M, Jose A, Chipman P, Bhattacharya N, Daneshparvar N, McKenna R, et al. Completion of the AAV Structural Atlas: Serotype Capsid Structures Reveals Clade-Specific Features. Viruses. 2021;13(1).

27. Westhaus A, Cabanes-Creus M, Rybicki A, Baltazar G, Navarro RG, Zhu E, et al. HighThroughput In Vitro, Ex Vivo, and In Vivo Screen of Adeno-Associated Virus Vectors Based on Physical and Functional Transduction. Hum Gene Ther. 2020;31(9-10):575-589.

28. Wu Y, Han ZP, Duan MZ, Jiang LY, Tian TT, Jin DY, et al. Popularizing Recombinant Baculovirus-derived OneBac System for Laboratory Production of all Recombinant Adenoassociated Virus Vector Serotypes. Current Gene Therapy. 2021;21(2):167-176.

29. Wu Y, Jiang L, Geng H, Yang T, Han Z, He X, et al. A Recombinant Baculovirus Efficiently Generates Recombinant Adeno-Associated Virus Vectors in Cultured Insect Cells and Larvae. Mol Ther Methods Clin Dev. 2018;10:38-47.

30. Szczypka MS, Kwok K, Brot MD, Marck BT, Matsumoto AM, Donahue BA, et al. Dopamine production in the caudate putamen restores feeding in dopamine-deficient mice. Neuron. 2001;30(3):819-28.

31. Swanson LW. The projections of the ventral tegmental area and adjacent regions: a combined fluorescent retrograde tracer and immunofluorescence study in the rat. Brain Res Bull. 1982;9(1-6):321-53.

32. Phillipson OT and Griffiths AC. The topographic order of inputs to nucleus accumbens in the rat. Neuroscience. 1985;16(2):275-96.

33. Finch DM. Neurophysiology of converging synaptic inputs from the rat prefrontal cortex, amygdala, midline thalamus, and hippocampal formation onto single neurons of the caudate/putamen and nucleus accumbens. Hippocampus. 1996;6(5):495-512.

34. Groenewegen HJ, Wright CI, Beijer AV and Voorn P. Convergence and segregation of ventral striatal inputs and outputs. Ann N Y Acad Sci. 1999;877:49-63.

35. Britt JP, Benaliouad F, McDevitt RA, Stuber GD, Wise RA and Bonci A. Synaptic and behavioral profile of multiple glutamatergic inputs to the nucleus accumbens. Neuron. 2012;76(4):790-803.

36. Grahn JA, Parkinson JA and Owen AM. The role of the basal ganglia in learning and memory: neuropsychological studies. Behav Brain Res. 2009;199(1):53-60. 
37. Zingg B, Chou XL, Zhang ZG, Mesik L, Liang F, Tao HW, et al. AAV-Mediated Anterograde Transsynaptic Tagging: Mapping Corticocollicular Input-Defined Neural Pathways for Defense Behaviors. Neuron. 2017;93(1):33-47.

38. Madisen L, Zwingman TA, Sunkin SM, Oh SW, Zariwala HA, Gu H, et al. A robust and highthroughput Cre reporting and characterization system for the whole mouse brain. Nat Neurosci. 2010;13(1):133-40.

39. Simmons PA, Lemmon V and Pearlman AL. Afferent and efferent connections of the striate and extrastriate visual cortex of the normal and reeler mouse. J Comp Neurol. 1982;211(3):295-308.

40. Zingg B, Hintiryan H, Gou L, Song MY, Bay M, Bienkowski MS, et al. Neural networks of the mouse neocortex. Cell. 2014;156(5):1096-111.

41. Cai AL, Zheng N, Garth JT, Wu Y, Nie BB, Lin KZ, et al. Longitudinal neural connection detection using a ferritin-encoding adeno-associated virus vector and in vivo MRI method. Human Brain Mapping. 2021;42(15):5010-5022.

42. Wang Z, Maunze B, Wang Y, Tsoulfas P and Blackmore MG. Global Connectivity and Function of Descending Spinal Input Revealed by 3D Microscopy and Retrograde Transduction. J Neurosci. 2018;38(49):10566-10581.

43. Weiss AR, Liguore WA, Domire JS, Button D and McBride JL. Intra-striatal AAV2.retro administration leads to extensive retrograde transport in the rhesus macaque brain: implications for disease modeling and therapeutic development. Sci Rep. 2020;10(1):6970.

44. Mokhtar M and Singh P (2021) Neuroanatomy, Periaqueductal Gray. In StatPearls.

45. Reed JL, Pouget P, Qi HX, Zhou Z, Bernard MR, Burish MJ, et al. Widespread spatial integration in primary somatosensory cortex. Proc Natl Acad Sci U S A. 2008;105(29):102337.

46. Nakai J, Ohkura M and Imoto K. A high signal-to-noise $\mathrm{Ca}(2+)$ probe composed of a single green fluorescent protein. Nat Biotechnol. 2001;19(2):137-41.

47. Chen TW, Wardill TJ, Sun Y, Pulver SR, Renninger SL, Baohan A, et al. Ultrasensitive

48. Mohebi A, Pettibone JR, Hamid AA, Wong JT, Vinson LT, Patriarchi T, et al. Dissociable dopamine dynamics for learning and motivation. Nature. 2019;570(7759):65-70.

49. Azevedo FAC, Carvalho LRB, Grinberg LT, Farfel JM, Ferretti REL, Leite REP, et al. Equal Numbers of Neuronal and Nonneuronal Cells Make the Human Brain an Isometrically ScaledUp Primate Brain. Journal of Comparative Neurology. 2009;513(5):532-541.

50. Oberheim NA, Wang X, Goldman S and Nedergaard M. Astrocytic complexity distinguishes the human brain. Trends Neurosci. 2006;29(10):547-53.

51. Silver J and Miller JH. Regeneration beyond the glial scar. Nat Rev Neurosci. 2004;5(2):14656.

52. Bedbrook CN, Deverman BE and Gradinaru V. Viral Strategies for Targeting the Central and 
53. Wang D, Tai PWL and Gao G. Adeno-associated virus vector as a platform for gene therapy delivery. Nat Rev Drug Discov. 2019;18(5):358-378.

54. Suzuki K, Tsunekawa Y, Hernandez-Benitez R, Wu J, Zhu J, Kim EJ, et al. In vivo genome editing via CRISPR/Cas9 mediated homology-independent targeted integration. Nature.

55. Yang Y, Wang L, Bell P, McMenamin D, He Z, White J, et al. A dual AAV system enables the

56. Rajasethupathy P, Ferenczi E and Deisseroth K. Targeting Neural Circuits. Cell.

57. Urban DJ and Roth BL. DREADDs (designer receptors exclusively activated by designer drugs):

58. Marshall JD, Li JZ, Zhang Y, Gong Y, St-Pierre F, Lin MZ, et al. Cell-Type-Specific Optical

59. Kim CK, Yang SJ, Pichamoorthy N, Young NP, Kauvar I, Jennings JH, et al. Simultaneous fast

60. Van der Perren A, Van den Haute C and Baekelandt V. Viral vector-based models of Parkinson's

61. Deverman BE, Pravdo PL, Simpson BP, Kumar SR, Chan KY, Banerjee A, et al. Cre-dependent

62. Chan KY, Jang MJ, Yoo BB, Greenbaum A, Ravi N, Wu WL, et al. Engineered AAVs for

63. Liu D, Zhu M, Zhang Y and Diao Y. Crossing the blood-brain barrier with AAV vectors. Metab

64. Morabito G, Giannelli SG, Ordazzo G, Bido S, Castoldi V, Indrigo M, et al. AAV-PHP.B-

65. During DN, Dittrich F, Rocha MD, Tachibana RO, Mori C, Okanoya K, et al. Fast Retrograde Access to Projection Neuron Circuits Underlying Vocal Learning in Songbirds. Cell Rep. 2020;33(6):108364. 
67. Blanco-Suarez E, Caldwell AL and Allen NJ. Role of astrocyte-synapse interactions in CNS disorders. J Physiol. 2017;595(6):1903-1916.

68. Gleichman AJ and Carmichael ST. Astrocytic therapies for neuronal repair in stroke. Neurosci

69. Rama Rao KV, Curtis KM, Johnstone JT and Norenberg MD. Amyloid-beta inhibits

70. Bosson A, Boisseau S, Buisson A, Savasta M and Albrieux M. Disruption of dopaminergic

71. Jiang R, Diaz-Castro B, Looger LL and Khakh BS. Dysfunctional Calcium and Glutamate

72. Talantova M, Sanz-Blasco S, Zhang X, Xia P, Akhtar MW, Okamoto S, et al. Abeta induces

73. Maheshri N, Koerber JT, Kaspar BK and Schaffer DV. Directed evolution of adeno-associated

74. Perabo L, Endell J, King S, Lux K, Goldnau D, Hallek M, et al. Combinatorial engineering of

75. Kotterman MA and Schaffer DV. Engineering adeno-associated viruses for clinical gene therapy.

76. Wu P, Xiao W, Conlon T, Hughes J, Agbandje-McKenna M, Ferkol T, et al. Mutational analysis

77. Shi W, Arnold GS and Bartlett JS. Insertional mutagenesis of the adeno-associated virus type 2

78. Ried MU, Girod A, Leike K, Buning $\mathrm{H}$ and Hallek M. Adeno-associated virus capsids

79. Tan F, Chu C, Qi J, Li W, You D, Li K, et al. AAV-ie enables safe and efficient gene transfer to Rep. 2015;12(6):1056-68. 
81. Hudry E, Andres-Mateos E, Lerner EP, Volak A, Cohen O, Hyman BT, et al. Efficient Gene Transfer to the Central Nervous System by Single-Stranded Anc80L65. Mol Ther Methods Clin Dev. 2018;10:197-209.

82. Kelemen RE, Mukherjee R, Cao XF, Erickson SB, Zheng YA and Chatterjee A. A Precise Chemical Strategy To Alter the Receptor Specificity of the Adeno-Associated Virus. Angewandte Chemie-International Edition. 2016;55(36):10645-10649.

83. Horowitz ED, Weinberg MS and Asokan A. Glycated AAV Vectors: Chemical Redirection of Viral Tissue Tropism. Bioconjugate Chemistry. 2011;22(4):529-532.

84. Guenther CM, Kuypers BE, Lam MT, Robinson TM, Zhao J and Suh J. Synthetic virology: engineering viruses for gene delivery. Wiley Interdiscip Rev Nanomed Nanobiotechnol. 2014;6(6):548-58.

85. Katrekar D, Moreno AM, Chen G, Worlikar A and Mali P. Oligonucleotide conjugated multifunctional adeno-associated viruses. Sci Rep. 2018;8(1):3589.

86. Zhang C, Yao T, Zheng Y, Li Z, Zhang Q, Zhang L, et al. Development of next generation adenoassociated viral vectors capable of selective tropism and efficient gene delivery. Biomaterials. 2016;80:134-145.

87. Thadani NN, Yang JN, Moyo B, Lee CM, Chen MY, Bao G, et al. Site-Specific Posttranslational Surface Modification of Adeno-Associated Virus Vectors Using Leucine Zippers. Acs Synthetic Biology. 2020;9(3):461-467.

88. Pearce HA, Qian H, Connell TU, Huang D, Gottstein C, Donnelly PS, et al. Site-Specific Glycation and Chemo-enzymatic Antibody Sortagging for the Retargeting of rAAV6 to Inflamed Endothelium. Mol Ther Methods Clin Dev. 2019;14:261-269.

89. Chen YH, Keiser MS and Davidson BL. Adeno-Associated Virus Production, Purification, and Titering. Curr Protoc Mouse Biol. 2018;8(4):e56.

90. Fripont S, Marneffe C, Marino M, Rincon MY and Holt MG. Production, Purification, and Quality Control for Adeno-associated Virus-based Vectors. J Vis Exp. 2019(143).

91. Paxinos G and Franklin K. (2012) Paxinos and Franklin's the Mouse Brain in Stereotaxic Coordinates, Amsterdam: Elsevier/Academic Press. 
606

607

608

609

610

611

612

613

614

615

616

617

618

619

620

621

622

623

624

625

626

\section{Figure legends}

Fig.1 Natural serotype AAV11 capsid for efficient retrograde access to projection neurons

(A) Schematic diagram of virus injection, rAAV11-EF1 $\alpha$-EGFP-WPRE-pA $\left(3 \times 10^{9}\right.$ VG) were injected into caudate-putamen $(\mathrm{CPu})$ area in $\mathrm{C} 57 \mathrm{BL} / 6 \mathrm{~J}$ mice.

(B) The fluorescence distribution of EGFP expression in injection site $\mathrm{CPu}$. Scale bar $=1 \mathrm{~mm}$.

(C) The spread of the virus to the anterior side. Scale bar $=500 \mu \mathrm{m}$. (C1) Neurons were tagged in somatomor areas (MO) and anterior olfactory nucleus (AON). Scale bar $=100 \mu \mathrm{m}$.

(D) The spread of the virus to the posterior side, Scale bar $=1 \mathrm{~mm}$. (D1) Neurons were tagged in somatosensory area (SSp) and mediodorsal nucleus of thalamus (MD). Scale bar $=100 \mu \mathrm{m}$.

Fig.2 rAAV11 does not exhibit anterograde transsynaptic spread properties

(A) Connectivity diagram shows the injection site (primary visual cortex, V1) and connected upstream areas in superior colliculus $(\mathrm{SC})$ and caudate-putamen $(\mathrm{CPu})$, rAAV11-hSyn-Cre-WPRE-pA $\left(2 \times 10^{9} \mathrm{VG}\right)$ was performed in Ai14 transgenic mice, in which the expression of tdTomato fluorescent reporter is Cre-dependent.

(B) The tdTomato fluorescence expressed in injection site (V1). Scale bar $=1 \mathrm{~mm}$. (B1) No tdTomato fluorescence signal is detected in superior colliculus (SC) area, which is the downstream brain area of V1. Scale bar $=1 \mathrm{~mm}$.

(C) No tdTomato fluorescence signal is detected in caudate-putamen $(\mathrm{CPu})$ area, which is the downstream of V1. Scale bar $=200 \mu \mathrm{m}$ for $\mathrm{C}$ and $100 \mu \mathrm{m}$ for $\mathrm{C} 1$.

Fig.3 Retrograde infection tropism comparison of rAAV11 and rAAV2-retro injected into the $\mathrm{CPu}$

(A) Connectivity diagram shows the injection site (caudate-putamen, $\mathrm{CPu}$ ) and 
connected upstream areas in primary somatosensory area (SSp), somatomotor areas

(MO), orbital area (ORB), and mediodorsal nucleus of thalamus (MD), rAAV11EF1 $\alpha$-EGFP-WPRE-pA and rAAV2-retro-EF1 $\alpha$-mCherry-WPRE-pA $\left(3 \times 10^{9}\right.$ VG in total) was performed in $\mathrm{C} 57 \mathrm{BL} / 6 \mathrm{~J}$ mice.

(B) Fluorescence distribution of EGFP (rAAV11) and mCherry (rAAV2-retro) expression in injection site $\mathrm{CPu}$. Scale bar $=1 \mathrm{~mm}$. (B1) The fluorescence distribution of EGFP and mCherry expression in SSp. Scale bar $=150 \mu \mathrm{m}$.

(C) Fluorescence expression of neurons after virus infection in the anterior side of injection site. Scale bar $=1 \mathrm{~mm}$. Representative images reveal that the retrograde labeling patterns of rAAV11-EF1 $\alpha$-EGFP and rAAV2-retro-EF1 $\alpha$-mCherry are quite different in many regions, such as the $\mathrm{MO}(\mathrm{C} 1$, Scale bar $=150 \mu \mathrm{m})$ and ORB $(\mathrm{C} 2$, Scale bar $=150 \mu \mathrm{m})$.

(D) Fluorescence expression of neurons after virus infection in the posterior side of injection site. Scale bar $=1 \mathrm{~mm}$. Representative images reveal that the retrograde labeling patterns of rAAV11-EF1 $\alpha$-EGFP and rAAV2-retro-EF1 $\alpha$-mCherry are quite different in many regions, such as the SSp $(\mathrm{D} 1$, Scale bar $=150 \mu \mathrm{m})$ and MD

Fig.4 Retrograde infection tropism comparison of rAAV11 and rAAV2-retro

(A) Connectivity diagram shows the injection site (ventral hippocampus, vHPC) and connected upstream areas in hippocampal field CA3 (CA3), nucleus of reuniens (RE), endopiriform nucleus dorsal part (EPd), lateral dorsal nucleus of thalamus (LD), and medial septal complex (MSC), rAAV11- EF1 $\alpha$-EGFP and rAAV2-retroEF $1 \alpha$-mCherry viruses were mixed at the particle ratio of $1: 1\left(3 \times 10^{9} \mathrm{VG}\right.$ in total $)$ and were injected into vHPC of C57BL/6J mice.

(B) Fluorescence distribution of EGFP (rAAV11) and mCherry (rAAV2-retro) expression in injection site vHPC. Scale bar $=1 \mathrm{~mm}$. 
662 (C) Representative images reveal that the retrograde labeling patterns of rAAV11663 EF1 $\alpha$-EGFP and rAAV2-retro-EF1 $\alpha$-mCherry are quite different in many regions, 664 such as the CA3, RE, EPd, LD (C1, Scale bar $=150 \mu \mathrm{m})$ and MSC (C2, Scale bar 665 $=200 \mu \mathrm{m})$.

666

667

668

669

670

671

672

673

674

Fig.5 Retrograde infection tropism comparison of rAAV-Y and rAAV2-retro injected into the PAG

(A) Connectivity diagram shows the injection site (periaqueductal gray, PAG) and connected upstream areas in ectorhinal area (ECT) and ventromedial hypothalamic nucleus (VMH), rAAV11-EF1 $\alpha$-EGFP and rAAV2-retro-EF1 $\alpha$-mCherry viruses were mixed at the particle ratio of $1: 1\left(1.8 \times 10^{9} \mathrm{VG}\right.$ in total $)$ and were injected into PAG of C57BL/6J mice.

(B) Fluorescence distribution of EGFP (rAAV11) and mCherry (rAAV2-retro) expression at injection site PAG. Scale bar $=200 \mu \mathrm{m}$.

(C) EGFP (rAAV11) and mCherry (rAAV2-retro) expression in ECT. Scale bar $=100$ $\mu \mathrm{m}$.

(D) EGFP (rAAV11) and mCherry (rAAV2-retro) expression in VMH. Scale bar $=100$ $\mu \mathrm{m}$.

\section{Fig.6 Use the rAAV11 to trace the upstream input network of the SSp}

(A) Connectivity diagram shows the injection site (primary somatosensory area, SSp) and connected upstream areas in ventral anterior-lateral complex of the thalamus (VAL) and ectorhinal area (ECT), rAAV11-hSyn-Cre $\left(2 \times 10^{9} \mathrm{VG}\right)$ was performed in Ail4 transgenic mice, in which the expression of tdTomato fluorescent reporter is Cre-dependent.

(B) The tdTomato fluorescence expressed in injection site (SSp). Scale bar $=500 \mu \mathrm{m}$.

(C) The tdTomato fluorescence expressed in upstream areas such as VAL and ECT. Scale bar $=100 \mu \mathrm{m}$. 


\section{$690 \quad$ Fig.7 rAAV11 can be used for analyzing functional network}

691 (A) Experimental injection paradigm for functional verification of the afferents from 692 the ventral tegmental area (VTA) to the nucleus accumbens (NAc), rAAV11-hSyn693 Cre $\left(2 \times 10^{9} \mathrm{VG}\right)$ was injected in NAc and rAAV9-DIO-GCaMP6m $\left(2 \times 10^{9} \mathrm{VG}\right)$ 694 in VTA, optical fiber for calcium ion signal detecting was implanted in VTA.

695 (B) The experimental set up.

696 (C) The GCaMP6m fluorescence expressed in VTA. Scale bar $=200 \mu \mathrm{m}$.

697 (D) Behavior-triggered average of $\Delta \mathrm{F} / \mathrm{F}$ signals.

698 (E) A representative trace of GCaMP6m $\Delta \mathrm{F} / \mathrm{F}$ signal with black arrows indicating 699 when the animal licked the sucrose solution.

(A) Schematic diagram of virus injection, rAAV11-GfaABC ${ }_{1}$ D-EGFP $\left(2 \times 10^{9} \mathrm{VG}\right)$ virus was injected into dentate gyrus (DG) area of C57BL/6J mice.

(B) EGFP expression in injection site DG with anti-GFAP indicating astrocytes. Scale bars $=200 \mu \mathrm{m}$ for $\mathrm{B}$ and $100 \mu \mathrm{m}$ for $\mathrm{B} 1$. 
708 Fig.1 Natural serotype AAV11 capsid for efficient retrograde access to projection 709 neurons

A

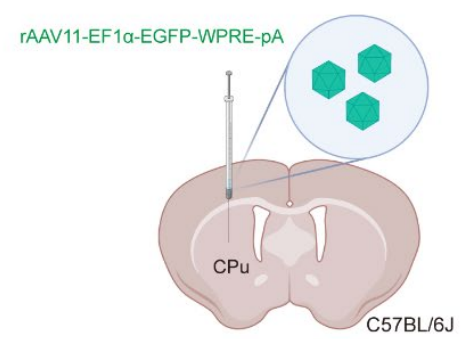

$\mathbf{B}$

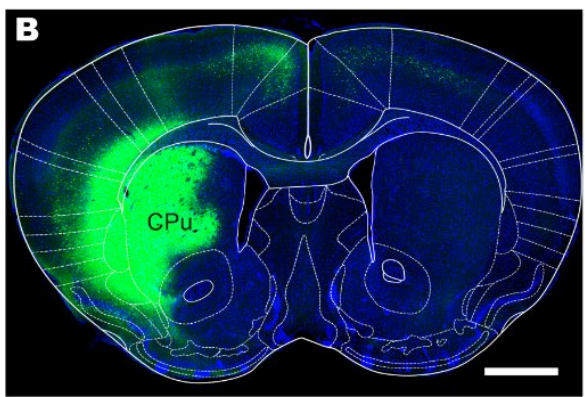

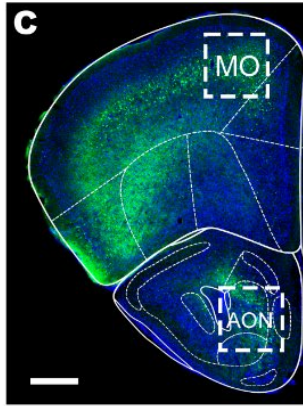

C1 DAPI

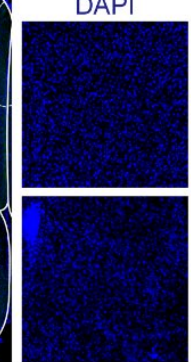

EGFP
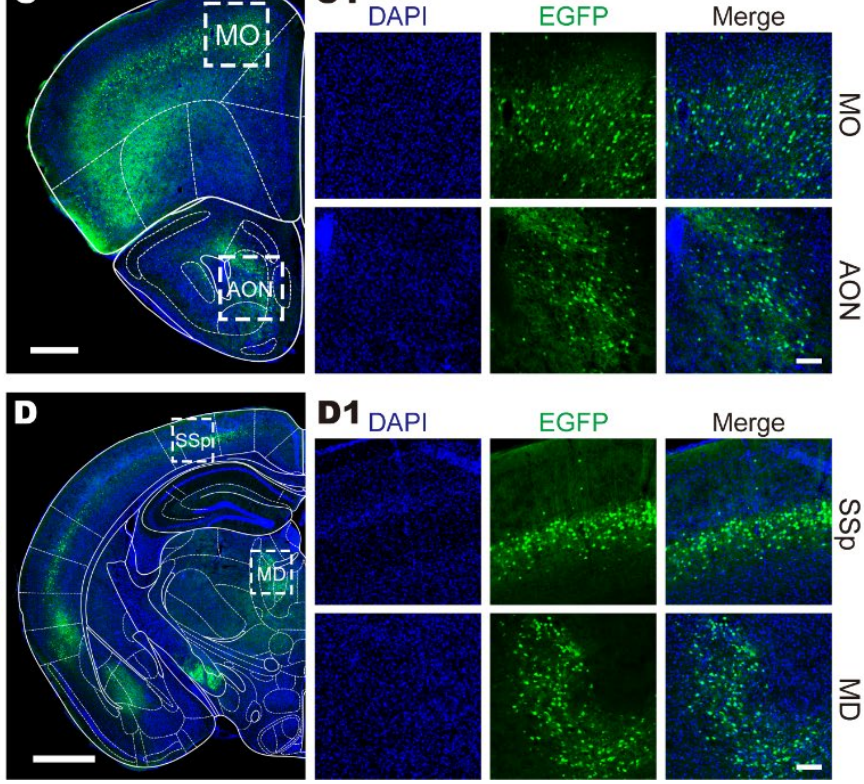

D1 DAPI

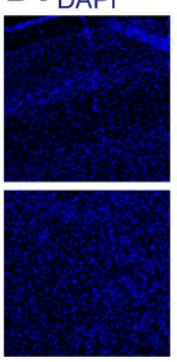

EGFP

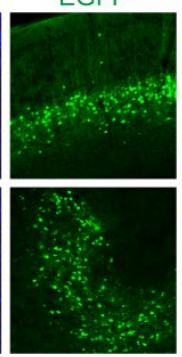

Merge

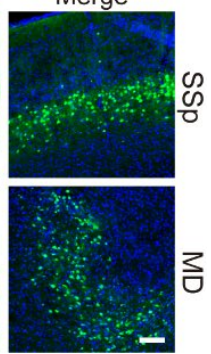

711

712

713

714

715

716

Fig.2 rAAV11 does not exhibit anterograde transsynaptic spread properties

A
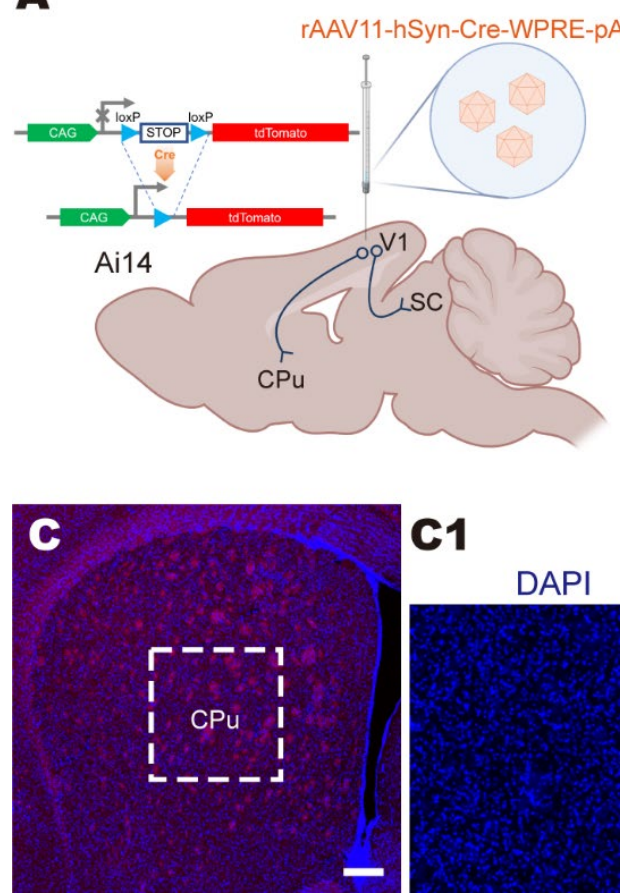

C1

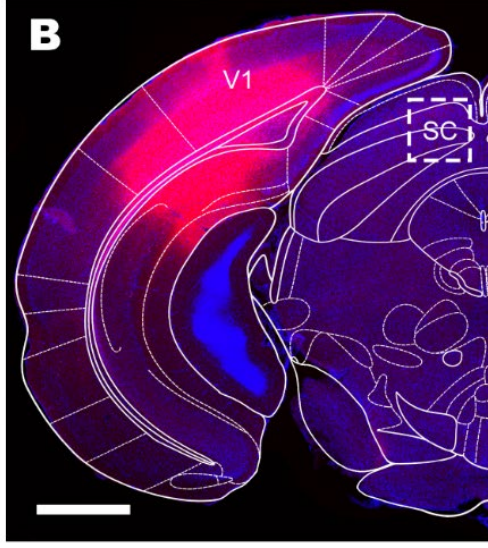

B1
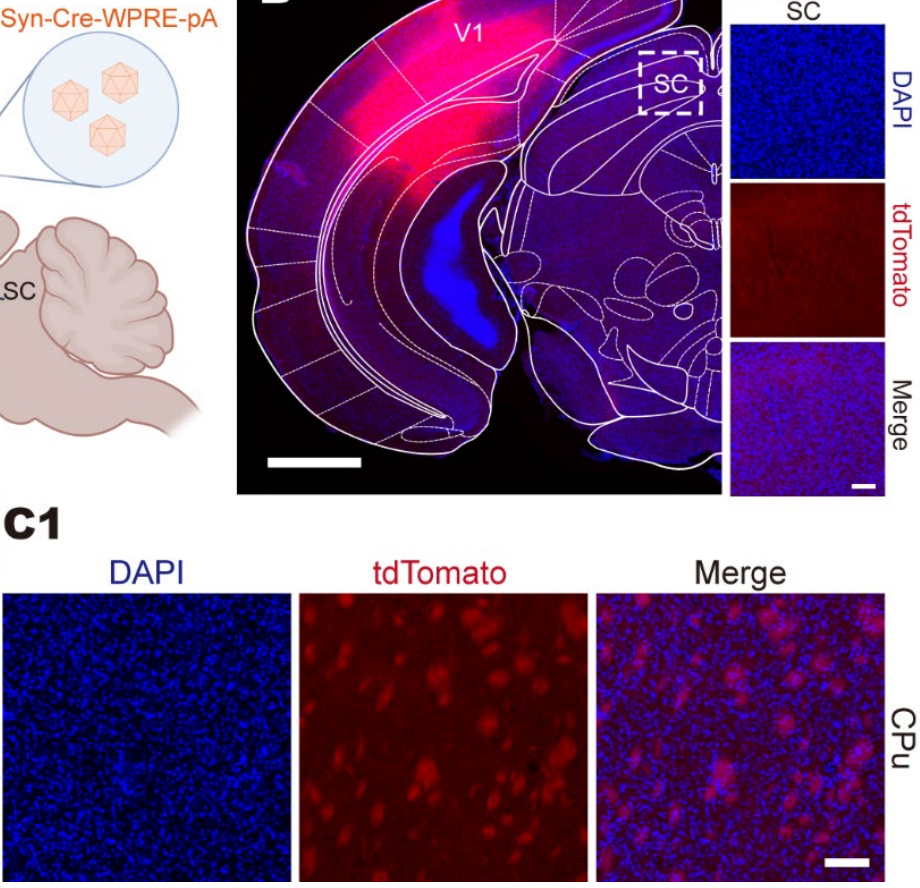

tdTomato

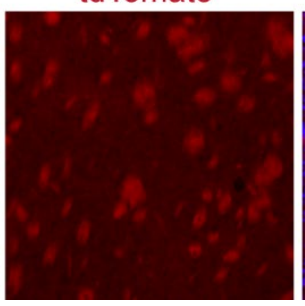

Merge

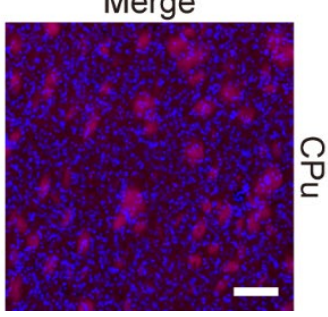


718 Fig.3 Retrograde infection tropism comparison of rAAV11 and rAAV2-retro injected 719 into the $\mathrm{CPu}$
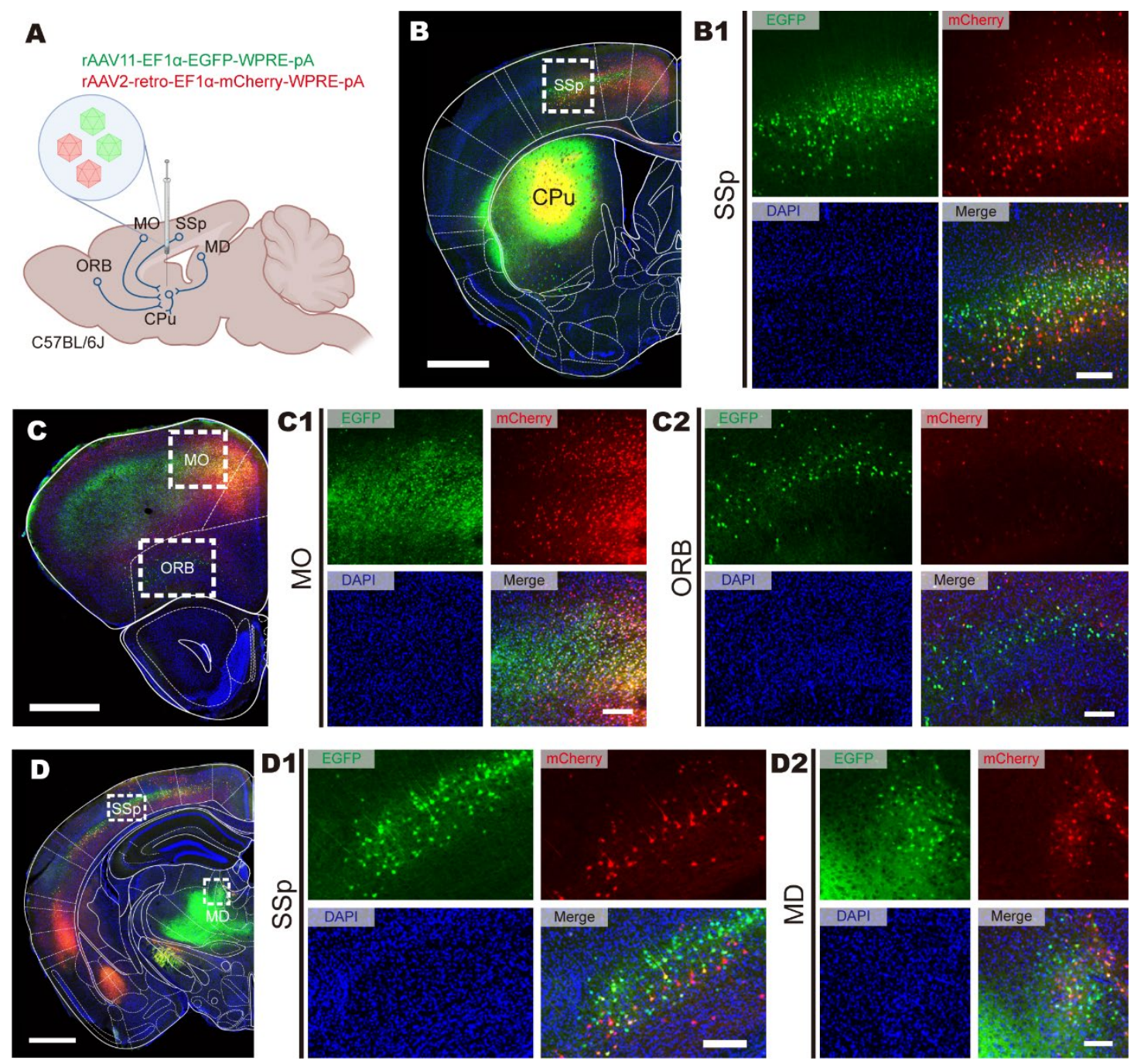

Fig.4 Retrograde infection tropism comparison of rAAV11 and rAAV2-retro injected 723 into the vHPC

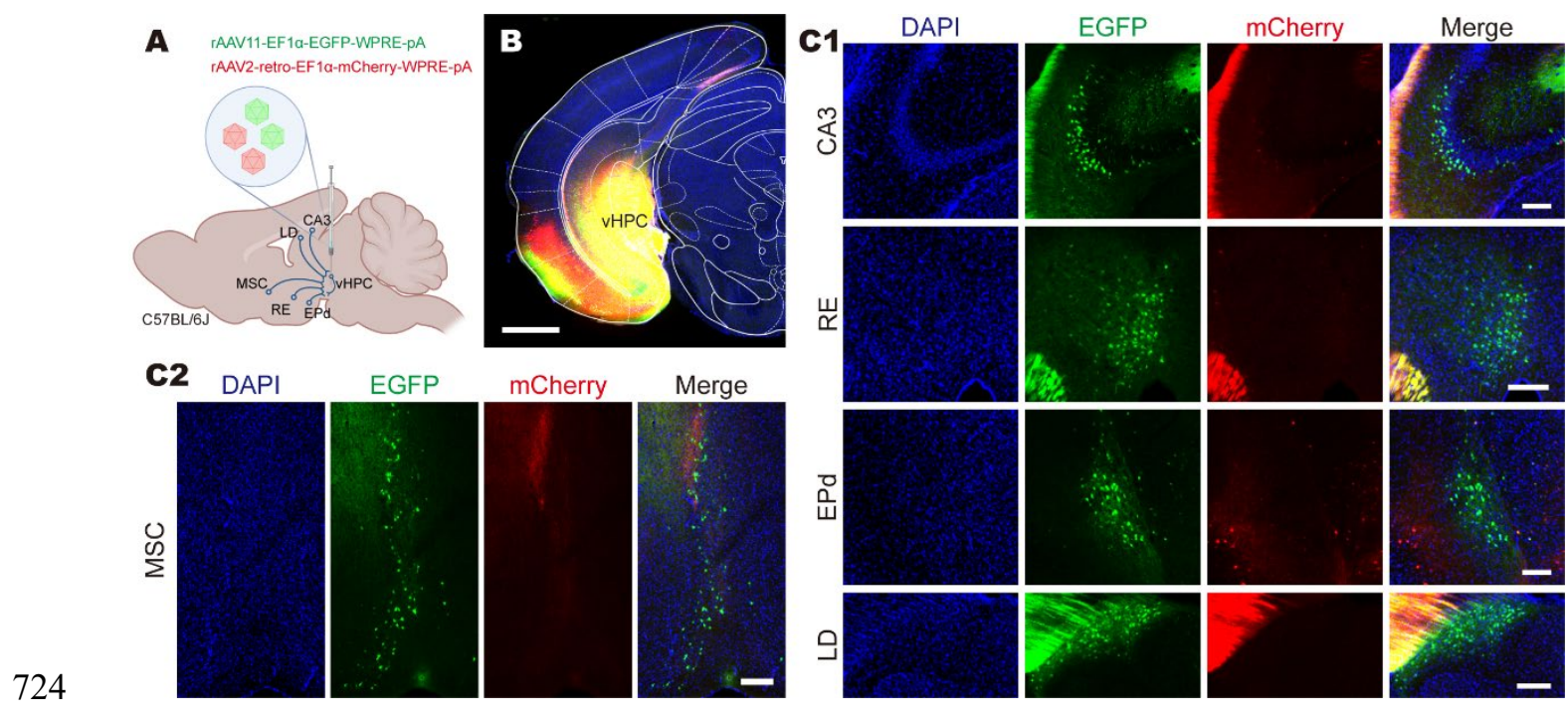


725 Fig.5 Retrograde infection tropism comparison of rAAV11 and rAAV2-retro injected 726 into the PAG

A

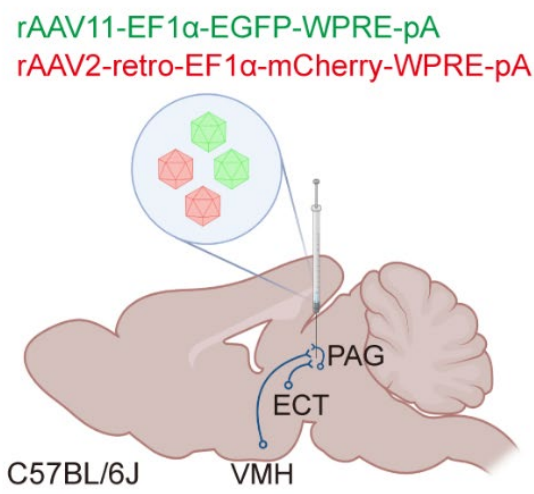

C ECT
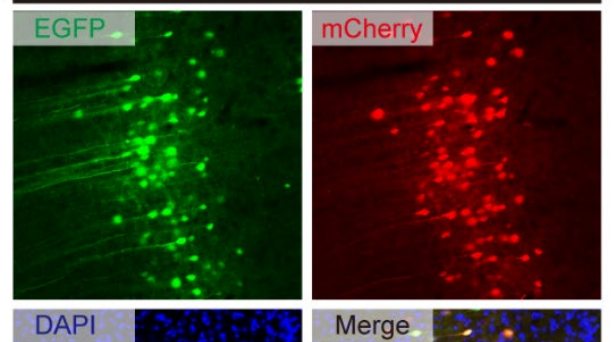

B.
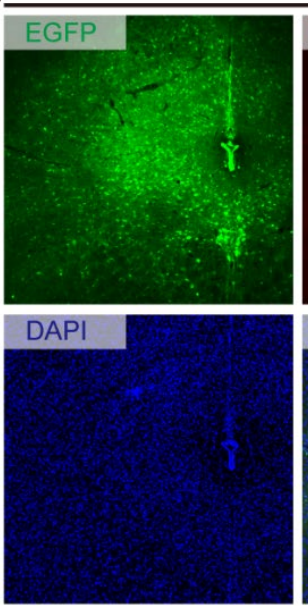

PAG

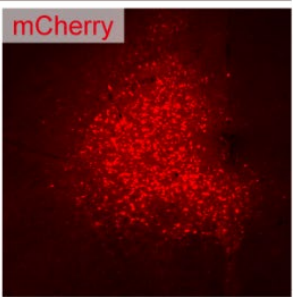

Merge

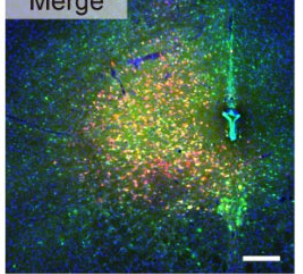

D

$\mathrm{VMH}$

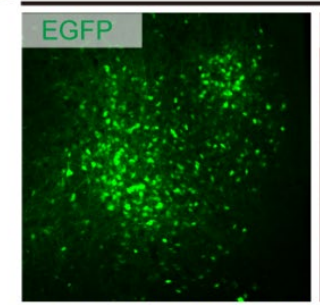

DAPI

mCherry

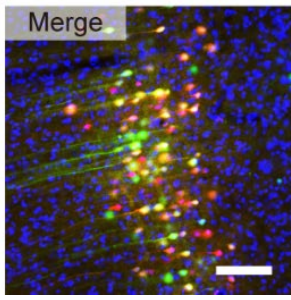

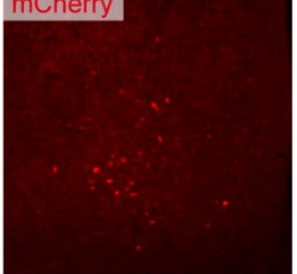

Merge

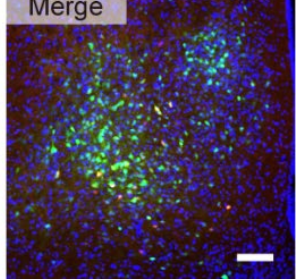

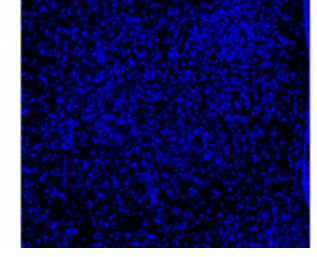


745 Fig.6 Use the rAAV11 to trace the upstream input network of the SSp
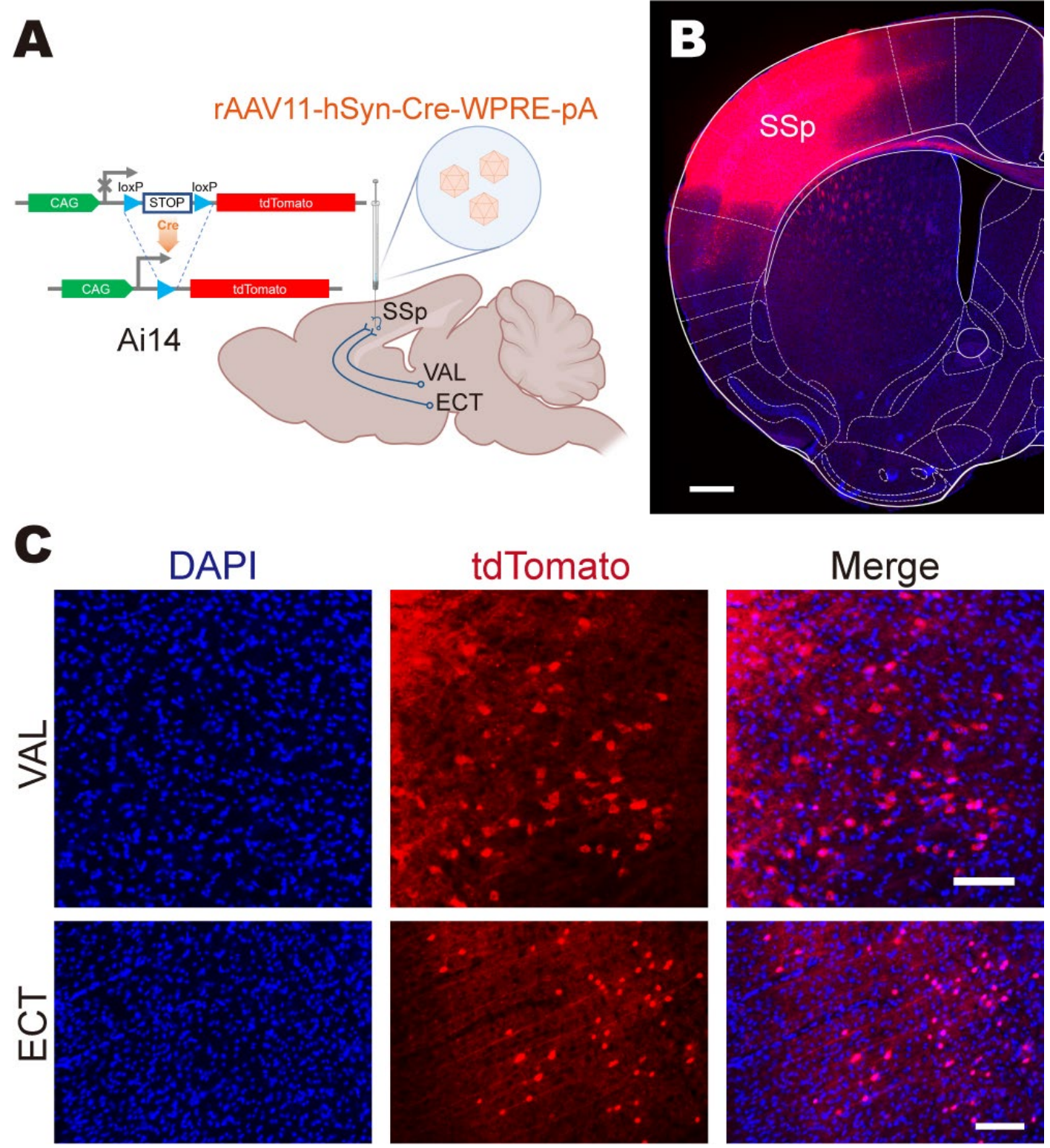
761 Fig.7 rAAV11 can be used for analyzing functional network
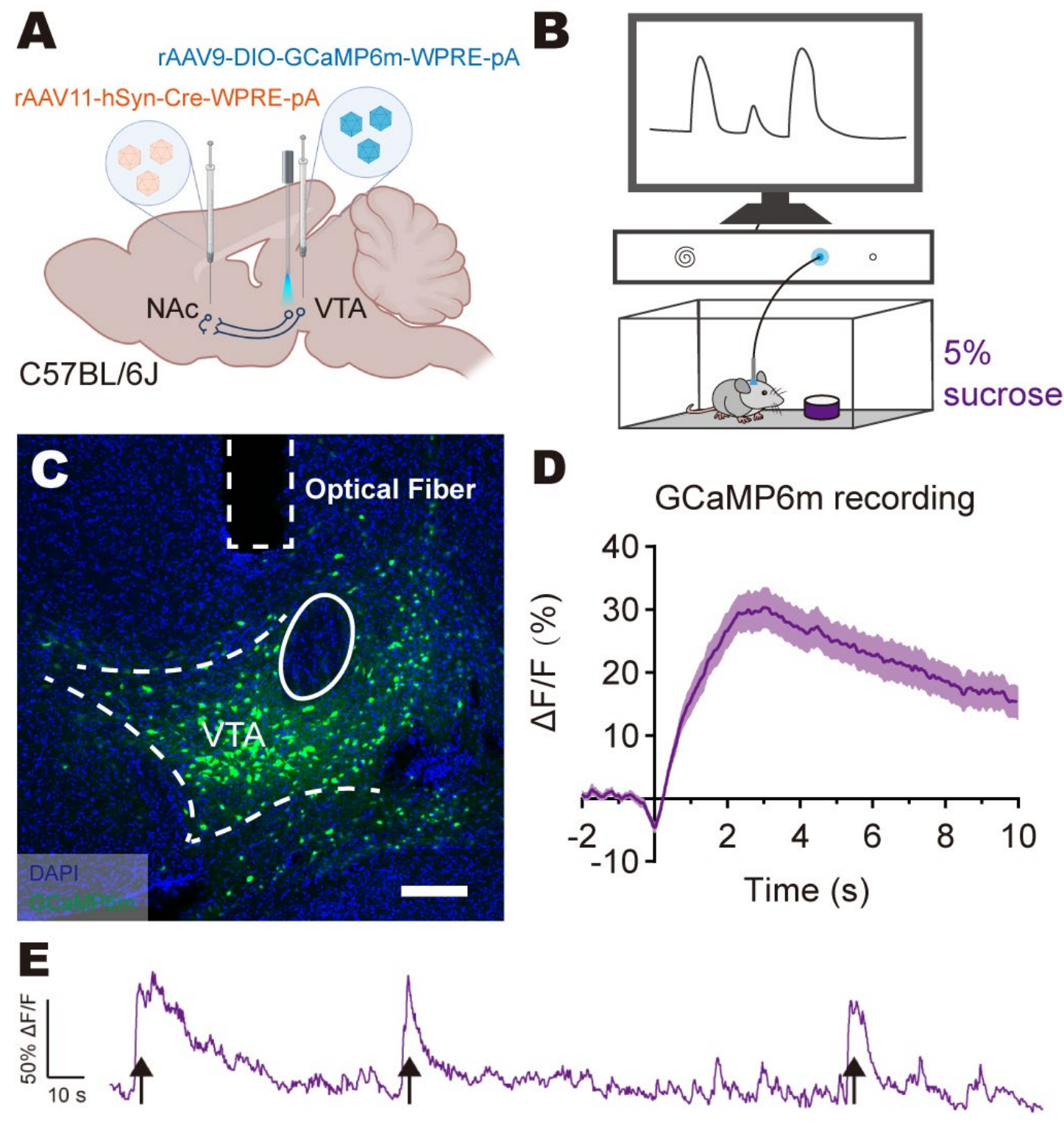
bioRxiv preprint doi: https://doi.org/10.1101/2022.01.13.476170; this version posted January 13, 2022. The copyright holder for this preprint (which was not certified by peer review) is the author/funder. All rights reserved. No reuse allowed without permission.

$778 \quad$ Fig.8 rAAV11 effectively target astrocytes

A

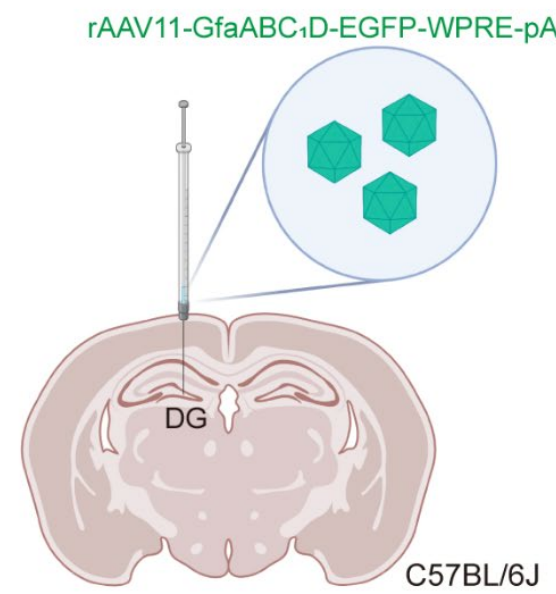

B1

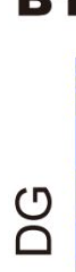

DAPI

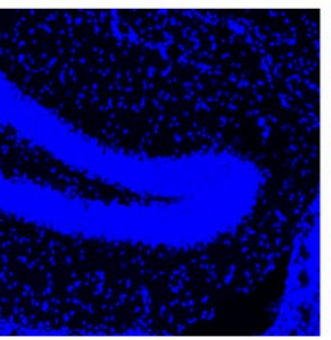

Anti-GFAP

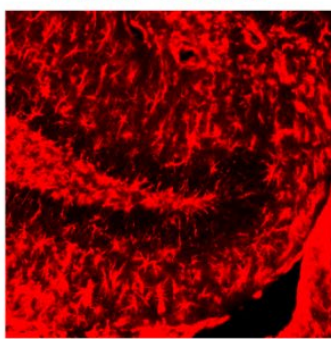

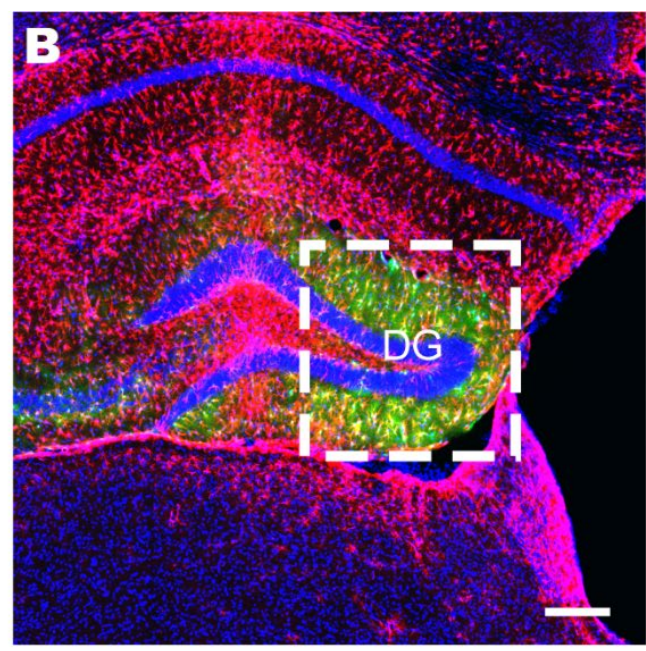

EGFP

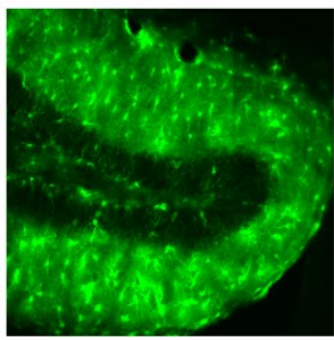

Merge

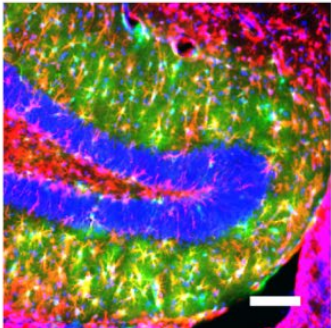

